\title{
Asymmetric surplus distribution and the price convergence path in Posted-Offer markets
}

\section{A note *}

\author{
Praveen Kujal \\ University of Arizona, Tucson AZ, USA
}

We report results from five Posted-Offer experiments with firm-specific quotas. The average contract price converges from below the competitive equilibrium. Thus, the independence of the contract price convergence path from surplus distribution is questioned in our results.

\section{Introduction}

Economic experiments have been useful in identifying certain characteristics of economic institutions. Subject to institutional rules certain behavioral characteristics have been observed and consistently replicated. For example, it is taken as given that Posted-Offer institutions have slow price convergence properties (towards the equilibrium) relative to the Double-Auction market institution [see Plott and Smith (1978) and Ketcham, Smith and Williams (1984)]. Another behavioral characteristic taken as given (after being replicated in several hundred experiments) is that average contracted prices in Posted-Offer (Posted-Bid) markets converge from above (below) the competitive equilibrium [see Plott (1982), Plott and Smith (1978), Ketcham et al. (1984)].

The direct investigation of the effect of surplus distribution on price convergence has not been done in many experiments. The only direct investigation of the effect of asymmetric surplus on average contract price convergence has been conducted by Davis and Williams (1986). Davis and Williams (henceforth DW) ran six Posted-Offer experiments. In half the experiments surplus distribution favored the sellers and in the other half the surplus distribution favored the buyers. DW report that, 'Posted-Offer contract prices tend to converge to the competitive equilibrium from above regardless of the distribution of the exchange surplus'. DW put forth evidence that suggests

Correspondence to: Praveen Kujal, Department of Economics, University of Arizona, Tucson, AZ 85721, USA.

* I would like to thank Arlington Williams for letting me use their experimental data. I would also like to thank Elizabeth Hoffman and Stan Reynolds for comments on an earlier draft. Funding for this paper was provided by Graduate Support Program of the Economic Science Laboratory. 

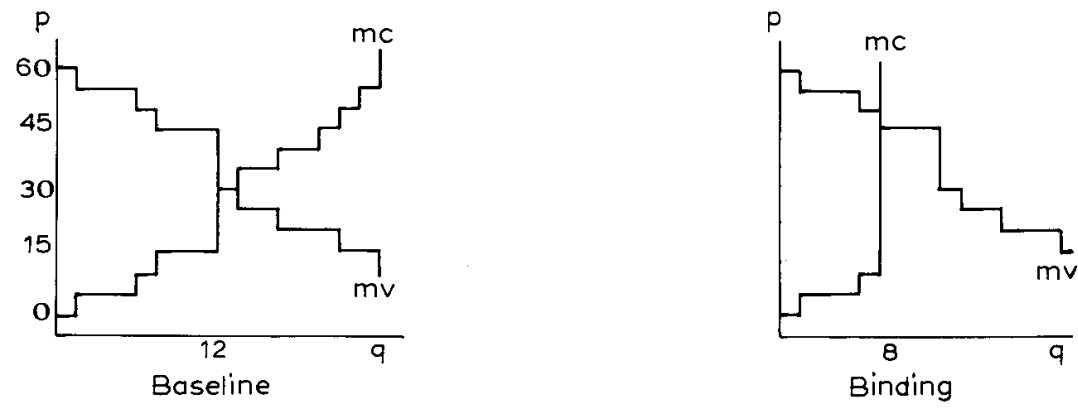

Fig. 1. Marginal cost and marginal valuations.

that institutional bias (characteristics ${ }^{1}$ ), such as the contract price convergence path, may dominate rent asymmetries.

In this paper we present evidence that violates DW's institutional bias conjecture. That is, the evidence suggests that the average contract price path may not be independent of the exchange surplus. We also suggest using average posted prices, rather than average contracted prices, to study the institutional bias conjecture (for the price convergence path).

The paper is organized as follows. In section 2 we look at the experimental design. In section 3 we discuss the results and provide arguments in favor of the alternative approach suggested. Section 4 is the conclusion.

\section{Experimental design}

The experiments use the Plato Posted-Offer exchange mechanism. We obtain asymmetric surplus distribution by using firm-specific binding quotas (bq) ${ }^{2}$. Surplus distribution is determined from the competitive outcome (ce) ${ }^{3}$. We get asymmetric surplus distribution favoring the seller

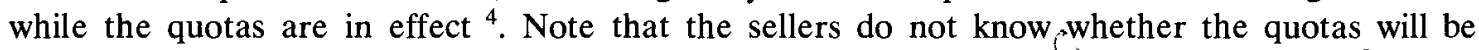
removed in the future. They only know that capacity is determined by a central authority ${ }^{5}$.

The buyers are simulated in the experiments, as the focus of the paper is to look at seller behavior. The buyers are perfect revealers and accept zero profit. The experimental design has four sellers.

The sellers get to see last periods posted prices. They have no.knowledge about the buyer marginal valuations, buyer behavior, or rivals costs. The sellers do not know that the buyers are simulated.

While the quotas are in effect each seller has a capacity of two units. All units can be traded at the competitive price. The capacity increases to five after the quotas are removed. Each seller can

1 We associate institutional bias with institutional characteristics.

2 This makes our design fundamentally different from other Posted-Offer designs. That is, asymmetric surplus distribution has been imposed exogenously by using binding quotas. The experimental design has symmetric distribution of surplus after the quotas are removed.

3 The price that clears the market.

4 For details on the experimental design see Kujal (1991).

5 For example, a central planner. 
price deviation



price deviation

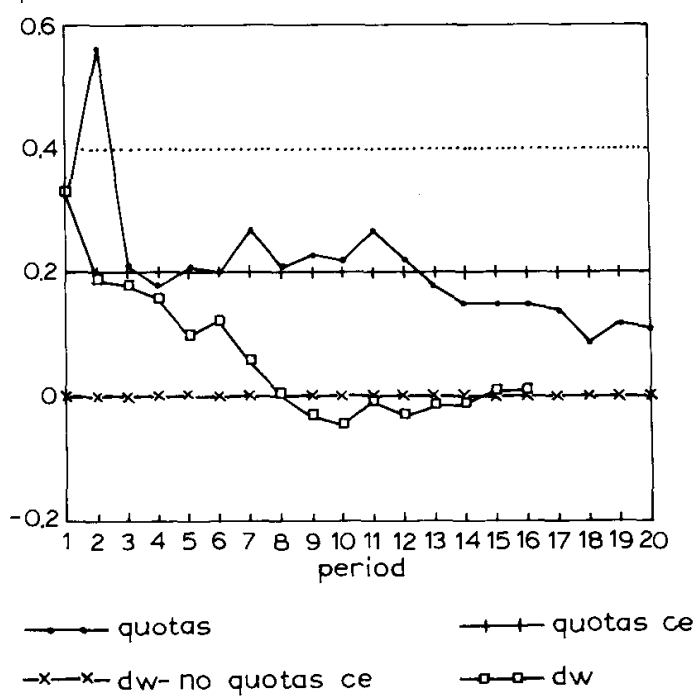

DW experiments ran for $15-16$ periods

Fig. 2.

trade three units at the competitive price. For supply-demand configurations see fig. 1. All units are expressed as deviations from the least cost units.

\section{Experimental results}

We report results from five experiments with binding quotas. The interesting result we get from the experiments is that the average contract price converges from below the competitive equilibrium. This result is unlike the average contract price convergence path observed in other PostedOffer experiments (with or without asymmetric distribution of surplus). In fig. 2 we present the contracted price plots for five experiments. We see that in all the experiments the average contracted price is below the CE (over the support $[5.45,5.50]$ ). We thus see that the convergence path may be affected by institutional characteristics, such as quotas, as well as by surplus distribution ${ }^{6}$.

Now, we suggest an alternative measure of institutional bias that reconciles our results with the DW and the other Posted-Offer experiment results. We suggest using average posted prices rather than average contracted prices. Using posted prices ${ }^{7}$ also makes it amenable to theoretical price predictions as any theory of non-cooperative prices predicts seller posted, rather than contracted, prices. Thus, posted prices should be a better indicator of the institutional properties relative to

6 Note, we otherwise refute the result in DW by using an extreme example here. Set total demand to be greater than total seller capacity. Assume constant marginal valuations and constant marginal costs. Here average contracted prices will always be less than or equal to the marginal valuations, or the $\mathrm{CE}$.

7 Davis and Holt (1991) uses median posted rather than average contracted price in their paper. 
contracted prices. Also, using posted prices we can reconcile our experimental results with the DW results. That is, using posted prices from the DW experiments we see that the prices converge from above the competitive equilibrium. Looking at fig. 2 we see that average posted prices in our experiments always converge from above the CE. We use average (rather than medium) prices as we want to capture the institutional bias as is reflected by (all of) the posted prices.

\section{Conclusion}

We thus see that DW's conjecture that institutional bias may dominate asymmetric surplus distribution is questioned by our experimental results. Average contract price convergence from below the equilibrium may be due to asymmetric surplus distribution or due to binding quotas. Whatever may be the cause, quotas or asymmetric surplus distribution, the dominance of institutional characteristics is questioned.

The results can, however, be reconciled by using posted, rather than contracted, prices as a reflection on the institutional characteristics. The posted prices (bids) would have an upward (downward) bias. But, the bias may be a true reflection of the institutional characteristics that we seek to understand and should, therefore, cause no problems.

\section{References}

Davis, D.D. and C.A. Holt, 1991, Capacity asymmetries, market power, and mergers in laboratory markets with posted prices, Working paper (University of Virginia, Charlottesville, VA).

Davis, D.D., C.A. Holt and A.P. Villamil, 1990, Supracompetitive prices and market power in posted offer auctions, BBER discussion paper (University of Illinois, Urbana, IL).

Davis, D.D. and A.W. Williams, 1986, Rent asymmetries in posted offer markets, Journal of Economic Behavior and Organization 7, 303-316.

Kujal, P., 1991, Firm-specific output limits in a posted-offer market: Distributive and efficiency effects, Working paper 92-15 (University of Arizona, Tucson, AZ).

Ketcham, J., V.L. Smith and A. Williams, 1984, A comparison of posted-offer and double auction pricing institutions, Review of Economic Studies 167, 595-614.

Plott, C.R., 1982, Industrial organization theory and experimental economics, Journal of Economic Literature 20, $1485-1527$.

Plott, C.R. and V.L. Smith, 1978, An experimental examination of two exchange institations, Review of Economic Studies $45,133-153$.

Smith, Vernon L., 1982, Microeconomic systems as an experimental science, American Economic Review 72, Dec. 923-955. 